\title{
Starting Process in a Large-Scale Shock Tunnel
}

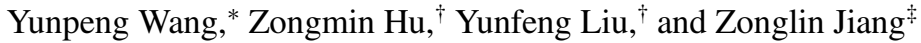 \\ State Key Laboratory of High-Temperature Gas Dynamics, \\ Chinese Academy of Sciences, 100190 Beijing, People's Republic of China
}

\section{DOI: $10.2514 / 1 . J 054145$}

\begin{abstract}
The starting process of the flow in an expansion nozzle (nominal Mach number 6) with an outlet diameter of $1.5 \mathrm{~m}$ and $8.9 \mathrm{~m}$ length, which is used for a large-scale hypersonic shock tunnel in the Key Laboratory of High-Temperature Gas Dynamics, was simulated and analyzed at incident Mach number $M_{s}=3.9$. The calculating domains include the driven section (the shock-tube end, about $4.8 \mathrm{~m}$ length), the nozzle with about $8.9 \mathrm{~m}$ length, and part of the test section (more than $3 \mathrm{~m}$ ). The characteristics of unsteady nozzle flow, including the shock wave patterns in the nozzle inlet region and inside the nozzle, were analyzed numerically in the viscous and inviscid flow regimes, respectively. The pressure and Mach number results were presented and discussed by comparing with the experimental findings, where the simulated results of the reflected shock wave in the shock tube and the transmitted shock wave inside the nozzle were found to agree well with the test data. Additionally, the case without a contraction section for the throat configuration was also calculated and compared with the case with a contraction section. The effect of the starting flow in these two cases on the flowfield uniformity is discussed in detail in this paper.
\end{abstract}

Nomenclature

$\begin{array}{ll}M_{s} & =\text { incident shock Mach number } \\ t & =\text { time, } \mathrm{s}\end{array}$

\section{Introduction}

$\mathbf{T}$ HE flow in a hypersonic shock tunnel starts when a strong planar incident shock wave reaches the nozzle inlet (the second diaphragm). The resulting flow is unsteady, and the gas behind the reflected shock wave at the shock-tube end wall is expanded to high flow Mach numbers in the nozzle. It evolves gradually toward a quasi-steady regime and a free expansion flow is established just downstream of the nozzle exit. The process, starting with the incident shock wave entering the nozzle until a quasi-steady flow is achieved, is called the starting process of the nozzle [1]. Aerodynamic measurements on models placed in the high-enthalpy freejet flow are generally of interest only after a quasi-steady flow has been established in the nozzle and in the test section. Therefore, understanding the unsteady starting process in the nozzle flow is very important to reducing the startup time. Smith [2] and Amann [3] experimentally investigated the unsteady nozzle flow using shadowgraph and interferometric flow visualization methods. Their findings reveal that the starting process involves a primary shock and a secondary shock that matches the pressures between the flow at the nozzle inlet and the flow just behind the primary shock. There is also a slip surface resulting from a Mach reflection. Igra et al. [4] performed inviscid simulations for the sharp inlet wedge tested by Amann [3]; Prodromou and Hillier [5] presented numerical simulations for a case where a shock wave of Mach 3 arrived at the inlet to a twodimensional planar nozzle having a half-angle of $15 \mathrm{deg}$ and a rounded contour of the inlet region, where these computations were extended by Tocarcik-Polsky and Cambier [6] to include viscous effects. Jacobs [7] and Saito and Takayama [1] computed the time-

Presented as Paper 2015-2291 at the 22th AIAA Computational Fluid Dynamics Conference, Dallas, TX, 22-26 June 2015; received 20 December 2014; revision received 19 August 2015; accepted for publication 5 December 2015; published online 10 March 2016. Copyright @ 2015 by the American Institute of Aeronautics and Astronautics, Inc. All rights reserved. Copies of this paper may be made for personal and internal use, on condition that the copier pay the per-copy fee to the Copyright Clearance Center (CCC). All requests for copying and permission to reprint should be submitted to CCC at www.copyright.com; employ the ISSN 0001-1452 (print) or 1533-385X (online) to initiate your request.

*Assistant Professor, Institute of Mechanics; wangyunpeng @imech.ac.cn. Member AIAA.

${ }^{\dagger}$ Associate Professor, Institute of Mechanics.

†Professor, Institute of Mechanics. Associate Fellow AIAA. dependent flowfield with a full Navier-Stokes solver. Mouronval and Abdellah [8] investigated the numerical study on the transient flows developing in a supersonic nozzle using a high-accuracy scheme (i.e., fifth-order WENO) and analyzed the complex evolution of the flow. Candler and Perkins [9] discussed a design technique based on the Navier-Stokes equations, including the effects of turbulent vibrational nonequilibrium on axisymmetric hypersonic nozzle design. Candler [10] proposed a computational method that uses an excluded volume equation of state to represent high-pressure effects in the reservoir, for the simulation of hypersonic nozzles. The Spalart-Allmaras turbulence model with a compressibility correction is used to model the turbulent boundary layer on the nozzle wall and the simulations show good agreement with test data of Arnold Engineering Development Center Tunnel 9 and Calspan-University of Buffalo Research Center shock-tunnel facilities. Maclean et al. $[11,12]$ performed full unsteady Navier-Stokes simulations to study viscous effects in the large energy national shock tunnel facilities.

The present study performed the large-domain computation for a large-scale and long-test-duration detonation-driven shock tunnel, which has been set up in the Key Laboratory of High Temperature Gas Dynamics (LHD) of the Institute of Mechanics, China. This facility, which features high-enthalpy test flow of pure air, can duplicate most of the key flow parameters around a hypersonic vehicle flying from Mach 5 to 9 and altitude from 25 to $50 \mathrm{~km}$. During the nozzle starting process of an operation, the gas with high temperature and high pressure in the reservoir region generates a strong starting shock wave at the nozzle inlet once breaking the diaphragm located in the vicinity of the nozzle throat. During the shock-tunnel run, the starting flow significantly affects the largescale model tests in this huge facility, especially for aerodynamic force and heating measurements. Therefore, the detailed investigation, on the starting process of the nozzle flow, is necessary for developing the ground-based hypersonic test equipment and for evaluating their performance.

\section{Detonation-Driven Shock Tunnel}

The long-test-duration hypersonic detonation-driven shock tunnel [13] was developed based on the backward-running detonation driver with several innovative techniques by the end of May 2012, as shown by the photograph in Fig. 1 . The shock tunnel, JF12, is named after shock tunnels under the serial number in LHD. Its performance tests demonstrated that the facility is capable of reproducing the pure airflow with longer than $100 \mathrm{~ms}$ test duration, and it will be a useful tool for testing engine/frame-integrated hypersonic vehicles and investigating into fundamental physical issues in hypersonic and high-temperature gas dynamics. 


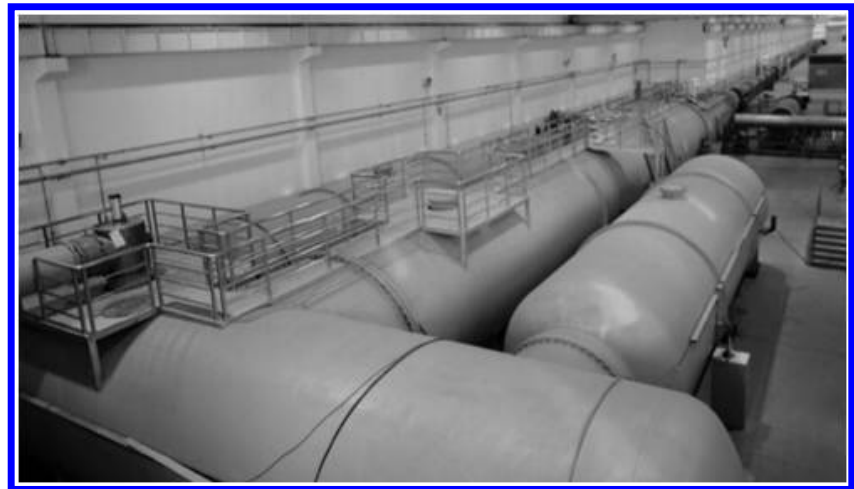

Fig. 1 Photograph of JF12 long-test-duration detonation-driven shock tunnel.

Figure 2 shows schematically the entire system of the JF12 hypersonic shock tunnel. From right to left, the first part is the vacuum system for damping wave reflection during the nozzle starting process, and its vacuum tank with a volume of $600 \mathrm{~m}^{3}$ is $50 \mathrm{~m}$ in length. The second part is the test section, which is $15 \mathrm{~m}$ in length and $3.5 \mathrm{~m}$ in diameter at the outlet. The contoured nozzle is $15 \mathrm{~m}$ in length and $2.5 \mathrm{~m}$ in diameter, and another nozzle is smaller, having an exit of $1.5 \mathrm{~m}$ in diameter for Mach number of 5-7, where the smaller one (nominal Mach number is 6) was employed for the numerical analysis in the present study. Next to the nozzle, there is the driven section, which is $89 \mathrm{~m}$ in length and $720 \mathrm{~mm}$ in diameter. The detonation driver is $99 \mathrm{~m}$ in length and $400 \mathrm{~mm}$ in diameter. The driver operates in the backward-running detonation mode, that is, the detonation is ignited at its right end. The detonation driver and the driven section are connected with the transition component by which the tube diameter is gradually reduced from 720 to $400 \mathrm{~mm}$. Between the detonation driver and the transition component, there is the diaphragm rig that is used to produce the incident shock wave in the shock tunnel after the direct detonation initiation. The damping section is located at the far left end of the facility and is $19 \mathrm{~m}$ in length and $400 \mathrm{~mm}$ in diameter. For experimental measurements, the JF12 shock tunnel is equipped with a 384-channel digital data acquisition system that is able to acquire, amplify, digitize, and store the data in real time. Immediately after each test run, the data are transferred to the computer in the control room for processing. Three sixcomponent force and moment balances are designed based on the 100 ms test duration, and calibration experimental data showed that the balances work pretty well.

\section{Numerical Methods}

\section{A. Governing Equations and Algorithm}

The two-dimensional axisymmetric, compressible Navier-Stokes and Euler equations were employed for the viscous and inviscid cases, respectively. The governing equations were discretized by the cell vertex finite volume method. The axisymmetric form of the Navier-Stokes equations is as follows [14]:

$$
\frac{\partial}{\partial t} \int_{\Omega} Q \mathrm{~d} \Omega+\oint_{\partial \Omega}\left(E_{c}-E_{v}\right) r \mathrm{~d} S=\int_{\Omega} Q^{*} \mathrm{~d} \Omega
$$

where $r$ is the coordinate in the radial direction, and the volume $\Omega$ is the product of the area of the control volume and an average radius $r$. The vector of the conservative variables consists of the four components

$$
Q=\left[\begin{array}{c}
\rho \\
\rho u \\
\rho v \\
\rho E
\end{array}\right]
$$

with $u$ and $v$ being the velocity components in the axial and radial directions, respectively. The vector of the convective fluxes is given by

$$
E_{c}=\left[\begin{array}{c}
\rho V \\
\rho u V+n_{x} p \\
\rho v V+n_{r} p \\
\rho H V
\end{array}\right]
$$

where $n_{x}$ is the unit normal vector in the axial direction, $n_{r}$ is the unit normal vector in the radial direction, and the contravariant velocity $V=n_{x} u+n_{r} v$. The vector of the viscous fluxes is defined as

$$
E_{v}=\left[\begin{array}{c}
0 \\
n_{x} \tau_{x x}+n_{r} \tau_{x r} \\
n_{x} \tau_{r x}+n_{r} \tau_{r r} \\
n_{x} \Theta_{x}+n_{r} \Theta_{r}
\end{array}\right]
$$

where

$$
\begin{gathered}
\Theta_{x}=u \tau_{x x}+v \tau_{x r}+k \frac{\partial T}{\partial x} \\
\Theta_{r}=u \tau_{r x}+v \tau_{r r}+k \frac{\partial T}{\partial r}
\end{gathered}
$$

are the terms describing the work of the viscous stresses and of the heat conduction in the fluid. Finally, the source term is as follows

$$
Q^{*}=\left[\begin{array}{c}
0 \\
0 \\
p-\tau_{\theta \theta} \\
0
\end{array}\right]
$$

The components of the viscous stress tensor are given by the relations

$$
\begin{aligned}
\tau_{x x} & =-\frac{2 \mu}{3} \nabla \cdot v+2 \mu \frac{\partial u}{\partial x} \\
\tau_{r r} & =-\frac{2 \mu}{3} \nabla \cdot v+2 \mu \frac{\partial u}{\partial r}
\end{aligned}
$$

$$
\begin{gathered}
\tau_{\theta \theta}=-\frac{2 \mu}{3} \nabla \cdot v+2 \mu \frac{v}{r} \\
\tau_{x r}=\tau_{r x}=\mu\left(\frac{\partial u}{\partial r}+\frac{\partial v}{\partial x}\right)
\end{gathered}
$$

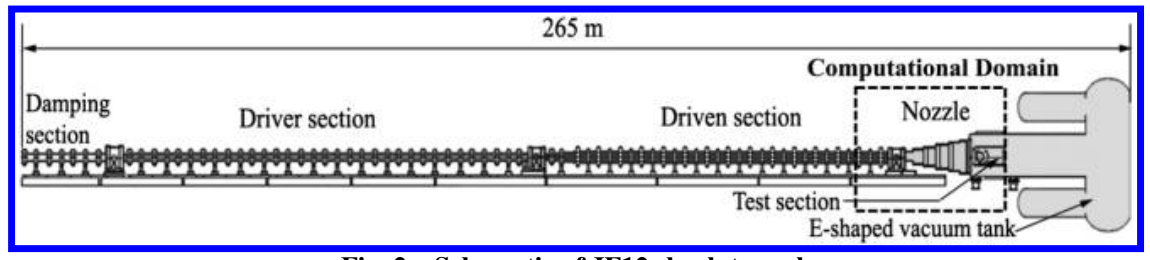

Fig. 2 Schematic of JF12 shock tunnel. 
with the divergence of the velocity

$$
\nabla \cdot v=\frac{\partial u}{\partial x}+\frac{\partial v}{\partial r}+\frac{v}{r}
$$

Additionally, Jameson and Baker's four stage Runge-Kutta method [15] was employed for time integration. Simple low-dissipation AUSM (SLAU) [16] and SLAU2 [17] schemes were used for calculating inviscid numerical fluxes. SLA U and SLAU2 are accurate, robust, and efficient for computations of wide-ranging Mach numbers. It hardly encounters shock anomalies, such as the carbuncle phenomena at high speeds. The MUSCL scheme with the van Albada flux limiter was adopted for the third-order-accurate numerical solutions. The viscous fluxes were calculated by the second-order central difference. The parallel implementation of the solver based on the MPI paradigm has been exploited to carry out a set of simulations for laminar and inviscid flows. The accuracy of the present in-house code is high enough, by the previous verification and validation, to capture all relevant flow features during the starting process of this huge test facility.

The nozzle expansion problem is a strange one because of the large range of Reynolds number scale in the problem. Candler [10] carried out the calculations of typical hypersonic nozzle flows and showed good agreement with measurable nozzle measurements in similar large-scale facilities using the Spalart-Allmaras one-equation turbulence model [18] with the Catris-Aupoix compressibility correction. Therefore, to evaluate the limitations without a turbulence model and the effects of turbulent boundary layer, a steady flow calculation was carried out using the Spalart-Allmaras turbulence model for the model A only.

\section{B. Model Configurations and Grid Generation}

Two computational models (see Fig. 3), the nozzle with the contraction section (model A, rounded inlet) and without a contraction section (model B, sharp edged inlet), were employed to examine the effects of the throat structure on flowfield uniformity in the test section.

The structured grid was used for all the numerical simulations. The grid consists of 13 blocks and the total number of grid points is approximately $3,120,000$, where each block was assigned to a single processor and contained $600 \times 400$ points. A grid convergence study was conducted with three different grid resolutions $(780,000$; $2,340,000$; and 3,120,000 grid points), and there was small change in the pressure and Mach distributions. However, the finest grid was employed for the higher resolution of the flowfield in such a large computational domain. To discuss the limitations of laminar flow, the finest grid was employed for the model A case in the steady turbulent calculation, and $y+$ value is less than one (actually, $y+\leq 0.972$ and its average value is about 0.534 ).

\section{Initial Conditions}

The flow domain employed in the computations included a 4.83 m-long section of the shock tube (part of driven section), a transition region into the throat (the contraction section with the length of $0.22 \mathrm{~m}$ ), an about 8.7-m-long expansion nozzle, and part of the test section. The initial conditions were chosen from the data obtained by

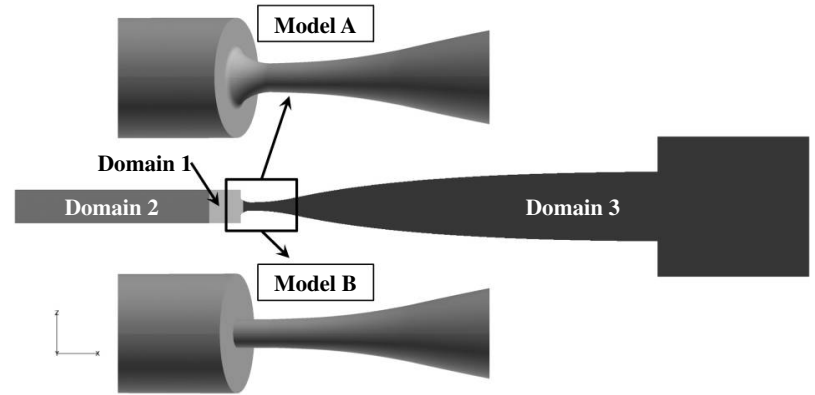

Fig. 3 Initial conditions for the different domains in CFD simulation.
Table 1 Initial and boundary conditions

\begin{tabular}{lrrr}
\hline \hline Conditions & Domain 1 & Domain 2 & Domain 3 \\
\hline Pressure, $\mathrm{Pa}$ & 27,000 & 474,615 & 10 \\
Temperature, K & 288 & 1,121 & 288 \\
Flow velocity, m/s & 0 & 1,051 & 0 \\
\hline \hline
\end{tabular}

a shock-tunnel calibration test. The nominal Mach number is 6 and test flow is the pure air. It is well known that the nozzle flow is extremely complex in a high-enthalpy facility. When the starting process is completed, the flow in the test section is the pure air during the test time and the flow temperature is lower due to the flow expansion in the nozzle. At this time, the real gas effects can be ignored in the test section. Therefore, as the preliminary study of the nozzle starting process, the simulation was simplified, where the chemical nonequilibrium effects were not considered in the present study based on the lower total temperature condition, and the test gas is frozen air with $\gamma=1.4$.

Initial conditions in the driven section (domain 1 in Fig. 3) were set to $P_{1}=27,000 \mathrm{~Pa}, T_{1}=288 \mathrm{~K}$, and $v_{1}=0 \mathrm{~m} / \mathrm{s}$; the postshock state (domain 2 in Fig. 3 ) of $P_{2}=474,615 \mathrm{~Pa}, T_{2}=112 \mathrm{~K}$, and $v_{2}=1051 \mathrm{~m} / \mathrm{s}$ was employed to the inflow plane. This resulted in an incident shock wave with $M_{s}=3.9$ and about $1300 \mathrm{~m} / \mathrm{s}$ shock wave speed.

Bursting of the secondary diaphragm (located at $x=0 \mathrm{~m}$ ) was modeled by running the simulation in two stages. In the first stage, the primary shock wave was allowed to propagate through to the throat, with the preshock conditions applied as initial conditions throughout the nozzle expansion. As soon as the primary shock reached the secondary diaphragm, the computation was stopped and the flow state in the nozzle and the test section (i.e., $x>0 \mathrm{~m}$, domain 3 in Fig. 3 ) was reset to $P_{n}=10 \mathrm{~Pa}, T_{n}=288 \mathrm{~K}$, and $v_{n}=0 \mathrm{~m} / \mathrm{s}$. The initial and boundary conditions are presented in Table 1 . A similar way to set the initial condition was discussed in Jacobs' study [7].

Some locations sketched in Fig. 4 are defined for storing the data of the pitot pressure (at $\mathrm{Pt}-1$ and $\mathrm{Pt}-\overline{2}$ ) and the stagnation pressure (at $P 5-1, P 5-2, P 5-3$, and $P 5-4)$, which was measured in the reservoir region.

In addition, the present unsteady simulations (pressure and Mach number) were compared with some test data. In the experiment, the test section Mach number is 6.1 and the stagnation conditions of $T_{o}=1900 \mathrm{~K}$ and $P_{o}=3.0 \mathrm{MPa}$ were employed to examine the present computational fluid dynamics (CFD) results.

\section{Results and Discussion}

Shadowgraph and schlieren techniques are used to visualize changes in density in the flowfields. The shadowgraph system captures second derivatives of flow density [19], whereas the schlieren system captures first derivatives. In this study, the numerical results were obtained using these two approaches. The following equation was employed to simulate the shadowgraph effects:

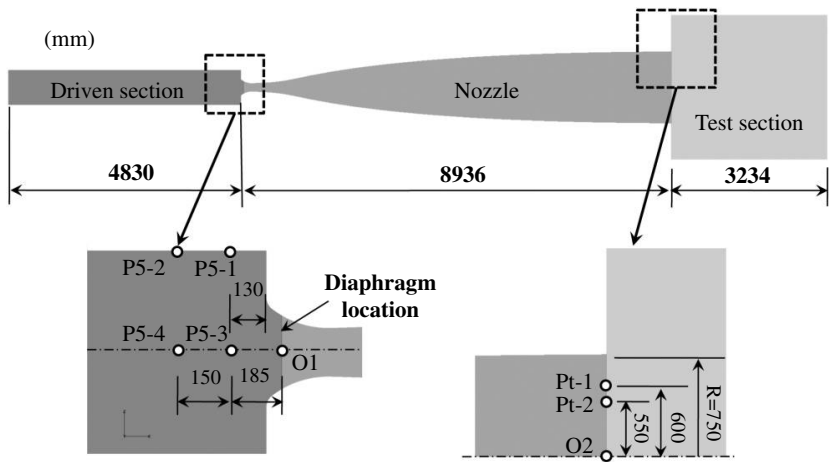

Fig. 4 The definition of locations for flow data in both the experiment and CFD (model A) 


$$
I=c \times\left(\frac{\partial^{2} \log (\rho)}{\partial x^{2}}+\frac{\partial^{2} \log (\rho)}{\partial y^{2}}\right)
$$

where $\rho$ is the flow density, $I$ is the light intensity, and $c$ is a constant, which depends on some parameters in the experiment.

\section{A. General Description of Physical Flow}

A typical wave diagram, for the detonation-driven shock tunnel operated in the backward-running detonation mode, is shown in Fig. 5. The detonation is initiated between the detonation driver and the driven section, and its wave graph is schematically presented in Fig. 5. The nozzle flow starts once the incident shock is reflected from the reservoir end and the reservoir state is interfered when the reflected expansion wave from the left end of the shock tunnel arrives.

In the present numerical study, the full-size model was used to understand the mechanism of the starting process in such a largescale detonation-driven shock tunnel. Obviously, the driven section is so long that the simulation was difficult to be run from the moment of $t=0 \mathrm{~ms}$ as defined in Fig. 5 . The initial states of the gas in regions 2 and 5 are matched to generate the incident shock, which is consistent with the test condition. Therefore, the effect of the interface shown in Fig. 5, on the startup time and the flow uniformity in the test section, was not discussed in the present study.

During the shock-tunnel run, once the secondary diaphragm bursts open ( $t=0 \mathrm{~ms}$, setting for the present simulation), the reflected shock (RS) is established in the shock tube. Simultaneously, some of the stagnant test gas is expanding through the throat and into the divergent section of the nozzle; the primary shock (PS) comes into being. As the PS travels down the nozzle it accelerates into the very low-pressure gas already in the nozzle. However, because of the diverging nozzle walls, it subsequently decelerates. Test gas, which accelerates through the nozzle throat following the PS, expands to a high Mach number. At the same time, the secondary shock (SS) is formed and is swept downstream through the nozzle. Figures 6 and 7 show the detailed evolution of the density field for the cases of models $\mathrm{A}$ and $\mathrm{B}$, respectively, using the numerical shadowgraph. Because of very low density in the nozzle and test section, the numerical shadowgraph technique was employed to show the flowfield more clearly. The propagation of the shock wave at the almost same transient for two models was compared during the range of $\mathrm{t} \approx 0.55-4.45 \mathrm{~ms}$. In the case of model $\mathrm{B}$, the strong interaction of the normal shocks with boundary layers occurring inside the nozzle throat gives rise to the shock train structures, which exist during the whole starting process. Based on the numerical result, it has a negative effect on the uniformity of the flowfield inside the nozzle and in the test section. The detailed description was carried out in the next section. On the contrary, the case of model A has no such pseudoshock phenomenon due to a contraction section, which also further accelerates the propagation of the PS and the test gas movement. Therefore, model A, which is employed in the JF12 shock tunnel, has better performance than model B for testing at $M \approx 6.0$ by the present unsteady simulation.

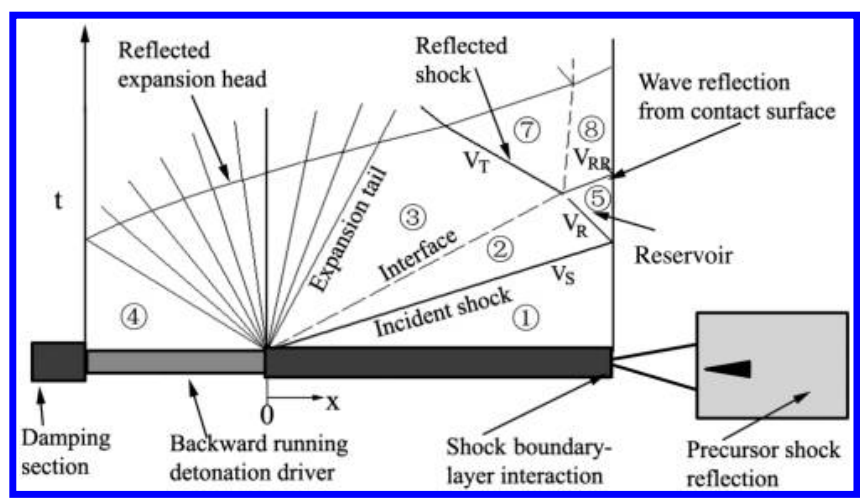

Fig. 5 Diagram $x$ - $t$ for a tailored running condition with the test time marked on it.
Figure $\underline{8}$ shows the evolution of the shock locations recorded on the axis in models A and B, respectively. Note that the speed of the PS in model A is faster than in the model B case. The RS becomes stronger due to the continuing reflected waves from the shock-tube end and the throat wall in the case of model A only. At the same time, the separation flow occurs on the shock-tube wall due to the negative pressure gradient and becomes large as time goes on. Figure 8 also shows the evolution of the separation point along the wall (a more detailed description can be found in the next section). Additionally, an obvious difference can be found that the speeds of the PS and SS in the case of model A are higher than that of model B, where the PS and the test gas in the case of model A are accelerated obviously due to a contraction structure.

\section{B. Shock Reflection Process in the Shock Tube}

\section{Reflected Shock}

The characteristics of the unsteady nozzle flow, including the shock wave patterns in the nozzle inlet region and inside the nozzle, were analyzed numerically in the viscous and inviscid flow regimes, respectively. In the driven section (shock tube), the flow separation occurs during the starting process on the shock-tube wall, and the flowfield becomes unsteady and more complicated. This mechanism of the interaction of RS with the boundary layer was studied in detail by Mark [20]. Figure 9 sketches Mark's model, which was also verified by the present numerical simulation.

Figures 10 and 11 show the detailed flow patterns and the shock structures in the shock reflection region (the shock-tube end), for models $\mathrm{A}$ and $\mathrm{B}$. To differentiate between the shock wave and the contact discontinuity (CD), a comparison was performed between the density and the pressure fields by the numerical schlieren. The results are shown at the almost same moment in cases A and B, respectively.

Figure 10a shows that the sequence begins at $t=0.239 \mathrm{~ms}$ with three RSs (i.e., RS1, RS2, and RS3 in the case of model A). RS1 is created and moved away from the end of the shock tube, whereas RS2 is generated based on the shock focusing along the nozzle axis, and RS3 is due to the reflection from the throat wall. Based on Mark's analysis [20], a complicated flow pattern is seen due to the interaction of RS and the boundary layer in the present CFD results. Actually, the flow separation region is becoming larger and larger during the nozzle flow startup. A main reason is that the RS continues to grow and become stronger due to the superimposition of RS1, RS2, and RS3, which means that these weak waves can combine to become a strong main reflected shock wave. At the same time, more RSs are generated from the throat wall. These phenomena can be seen in Fig. 10b. The numerical comparison in the density and pressure fields enables the shock structures to become clearer.

In model B, shown in Fig. 11, which is the case without the contraction section, the RS is created from the end wall of the shock tube only. A shock train phenomenon is generated inside the nozzle throat. It implies that the main RS is weaker and becomes slower than that of the model A case. Additionally, the separating region size of model B is smaller at the same moment as compared with the case of model A.

\section{Stagnation Pressure}

The pressure in the reservoir region (see Fig. 5) $P 5$ was discussed by comparing the CFD result with the test data obtained by the JF12 hypersonic shock tunnel. First, we focused on the initial stage of the starting process (i.e., the phase step of the $P 5$ signal marked by the box in Fig. 12).

In the experiment, two locations at the end of the driven section with an axial separation of $150 \mathrm{~mm}$ (see Fig. 4) were considered to measure the stagnation pressure in the reservoir region. The pressure history by experiment was depicted in Fig. 12 from which the physics of the flow inside the shock tube can be traced. Figure 13 shows the $P 5$ history at $P 5-1$ and $P 5-2$ during the starting process for the experiment and numerical simulation, respectively. The first jump $(t=0 \mathrm{~ms}$ at $P 5-2)$ represents the arrival of the incident shock wave, across which the pressure inside the driven section increases from 27 to around $470 \mathrm{kPa}$ at $P 5-2$. As the shock wave proceeds to the shock- 

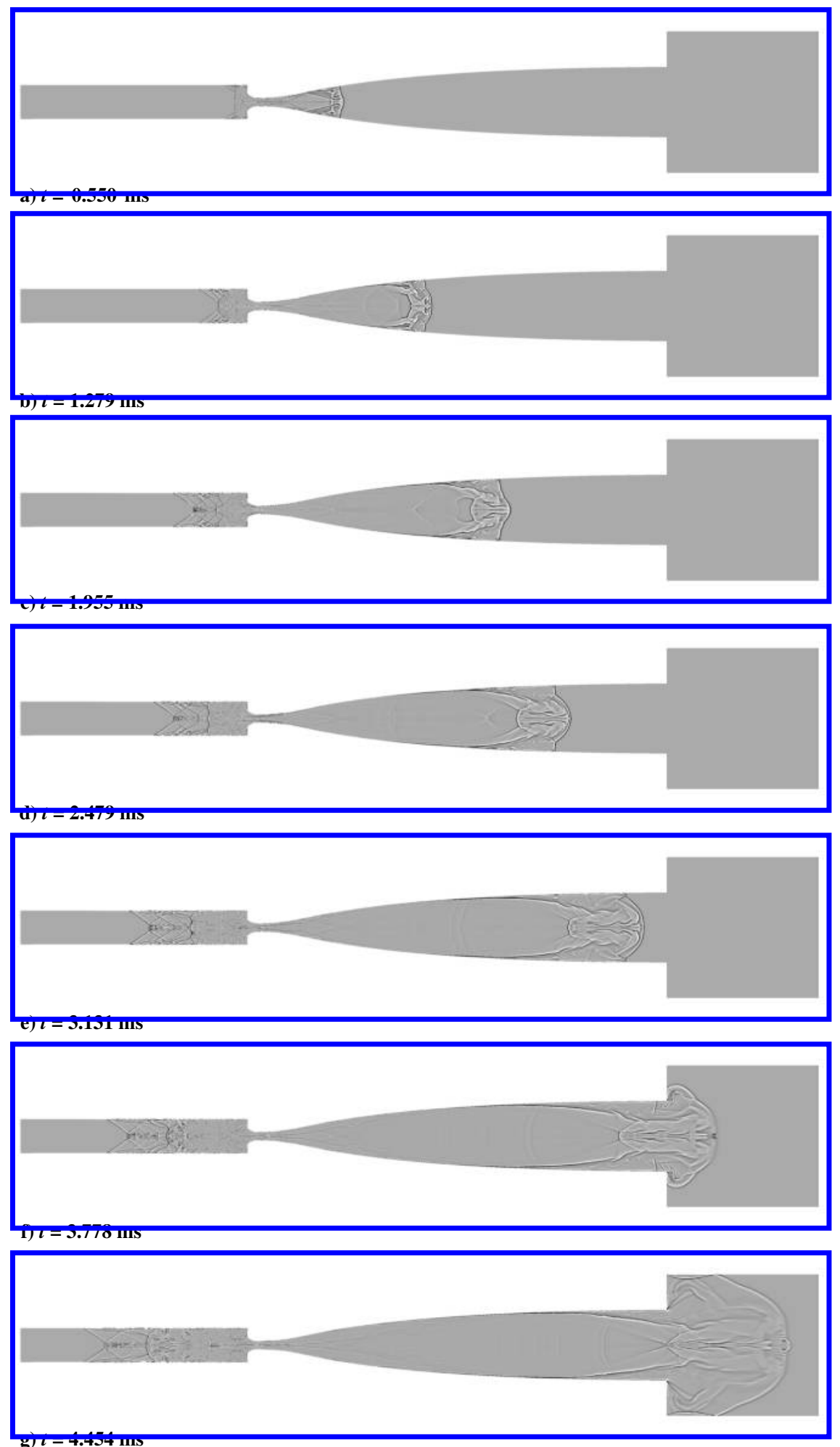

Fig. 6 Evolution of the density field by numerical shadowgraphs in the case of model $\mathrm{A}$.

tube end it will reflect and move in the opposite direction, increasing the pressure to nearly 3.0 MPa. Furthermore, the second jump of $P 5$ history obtained by experiment is generated by the separation shock (SEPS) along the wall, not by the reflected shock. These phenomena can also be seen directly in Fig. 10.

To compare with experiment, the numerical results (Figs. $13 \mathrm{~b}$ and $13 \mathrm{c})$ show good agreement with the test data. The shock wave speed $\overline{\text { can }}$ be determined from the first jumps of the pressure at $P 5-1$ and $P 5-2$. Because the distance between the two locations is known $(150 \mathrm{~mm})$, the time that the incident shock travels from $P 5-2$ to $P 5-1$ can be obtained from the pressure history shown in Fig. 13.
Therefore, the speed of the incident shock obtained by test is $1316 \mathrm{~m} / \mathrm{s}$, whereas the CFD result is $1293 \mathrm{~m} / \mathrm{s}$ in the case of the laminar flow, which indicates the percentage difference of about $1.7 \%$ from the test result.

However, some differences are also observed in the process of the shock reflection, especially the second jump obtained at both $P 5-1$ and $P 5-2$, which represents the RS passed through the locations $P 5-1$ and $P 5-2$, respectively, along the shock-tube wall. First, in the laminar simulation, the computational result shows the faster speed of the RS than the test case. The amplitude of the second jump is smaller in CFD simulation. These differences are attributed to the 


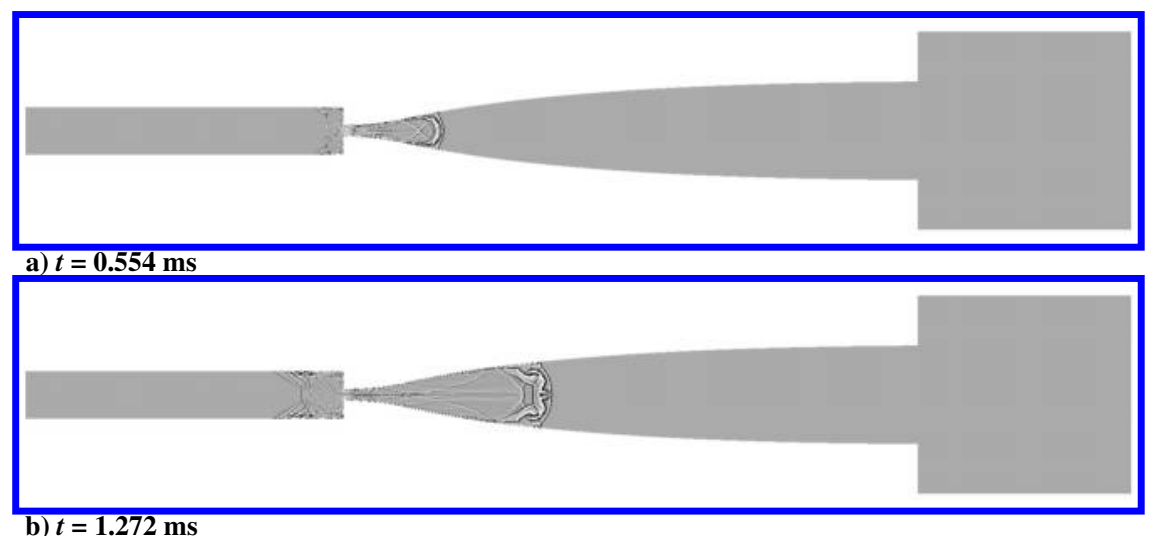

b) $t=1.272 \mathrm{~ms}$
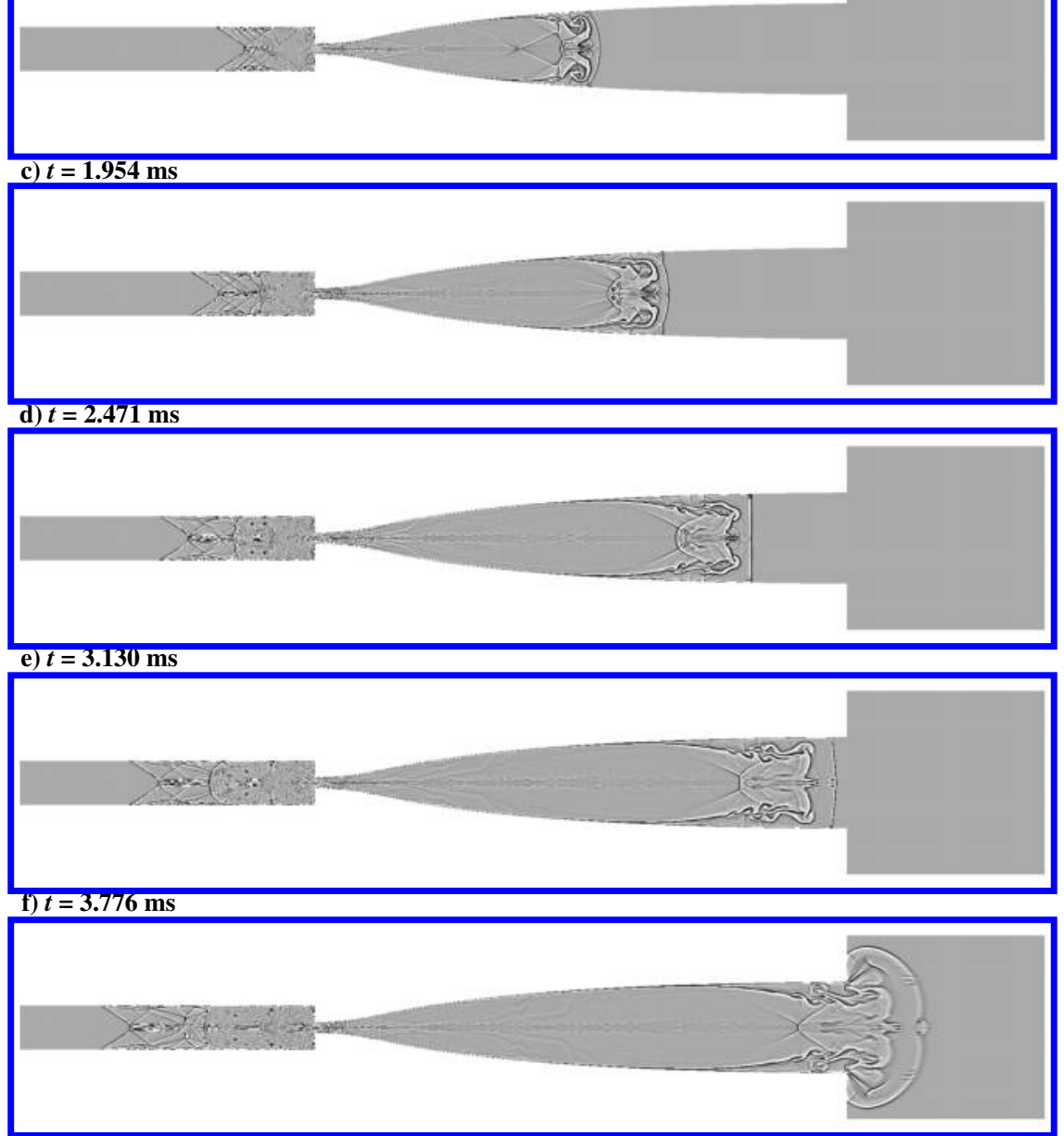

g) $t=4.452 \mathrm{~ms}$

Fig. 7 Evolution of the density field by numerical shadowgraphs in the case of model $B$.

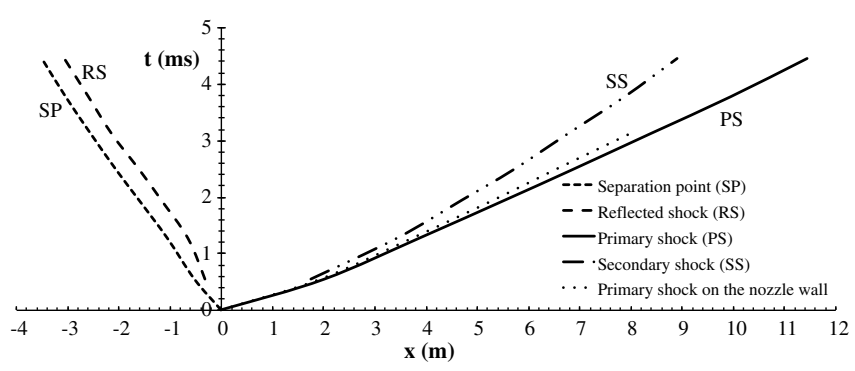

a) Model A

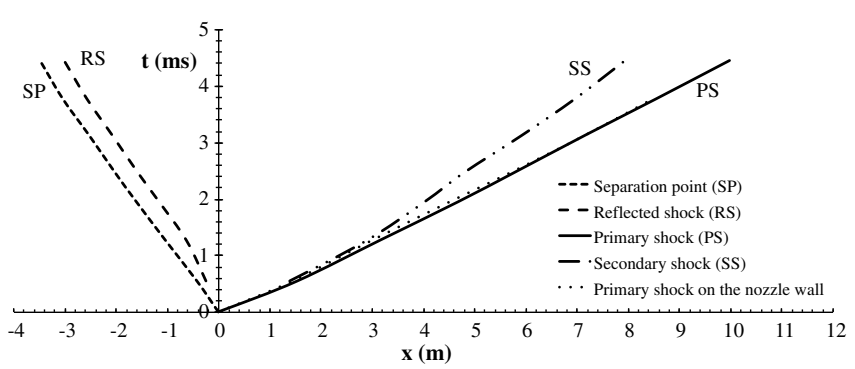

b) Model B

Fig. 8 Wave diagram $x$ - $t$ during the starting process. 
boundary-layer interaction and the effect of the diaphragm bursting. In the inviscid simulation, the shock speed, either of the incident shock wave or of the reflected shock wave, is closer to the experimental result, where the inviscid result shows the RS travel along the wall (no separation shock occurs). It indicates the following:

1) In the laminar simulation, RS propagation is not so fast, but the flow separation region becomes larger and larger as the shock wave moves away from the flat end of the shock tube, that is to say, the separating shock propagates more and more quickly with the increase of the separation region.

2) In the test case, the flow separation is not as large as in the shock tube, at least during the starting process; this can also explain that the moment of the third jump is closer in the case of the test measurement and the laminar simulation.

3) The more stronger interactions occur due to the thicker boundary layer; the present simulation has to pay no attention to the evolution of the boundary layer due to the large shock tube with more
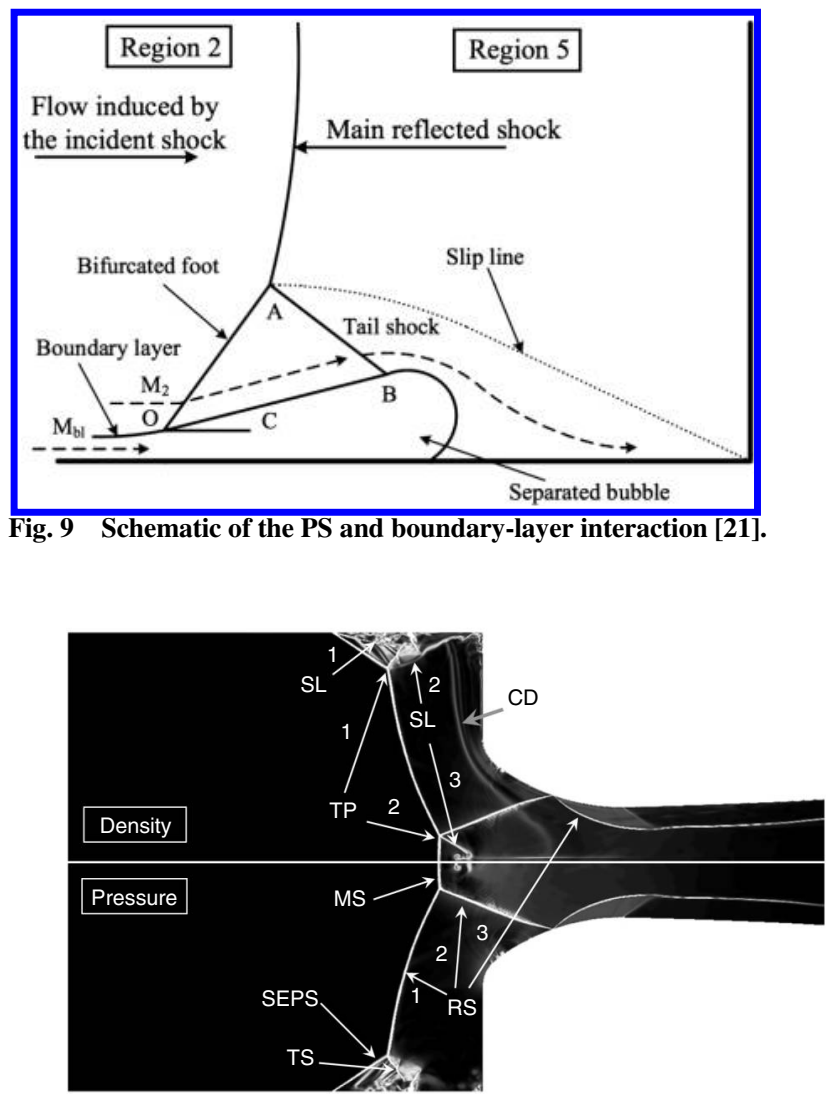

a) $t=0.239 \mathrm{~ms}$ than $80 \mathrm{~m}$ length (the driven section only). Therefore, the boundary layer (near the shock-tube end) is thicker in the test case, and the higher pressure value occurs during the second jump in the measurement data.

4) Figure $13 \mathrm{c}$ shows the more simple pressure curves, because there is no shock and boundary-layer interaction in the inviscid case.

\section{Starting Flow in the Nozzle}

The nozzle flow starts up at $t=0 \mathrm{~ms}$ with the PS propagating toward the throat. After that, the RS is established at the shock-tube end. Simultaneously, some of the stagnant test gas expands through the throat and into the divergent section of the nozzle. The complex structure of the shock waves evolves in the nozzle, and the effect of the boundary layer becomes larger on the transmitted shock, contact surface, and secondary shock system. These phenomena of the discontinuity were discussed in detail in this section, where they are shown in Fig. 14 and can also be seen in Figs. $\underline{6}$ and $\underline{7}$ for models A and B, respectively. In this section, model A with laminar flow was discussed basically due to the almost same flow pattern in the two models.

Figure 14 shows the flowfield at $t=1.191$ and $2.355 \mathrm{~ms}$, as time goes on, by the numerical schlieren graph. To further understand the shock structure (such as the shock wave and $\mathrm{CD}$ ), the density, pressure, and temperature fields were examined, respectively. Obviously, CD is observed clearly. The slip line (SL) near the nozzle wall indicates that the interaction of the boundary layer and the PS

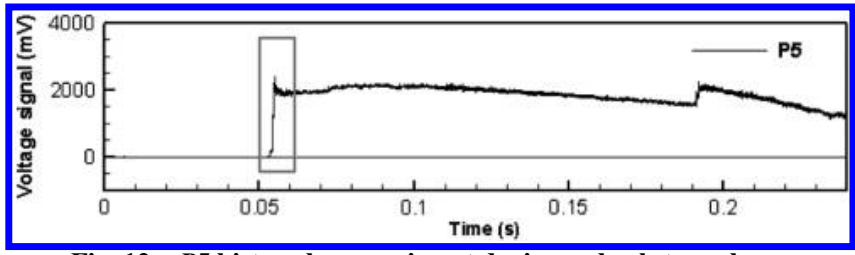

Fig. $12 P 5$ history by experiment during a shock-tunnel run.

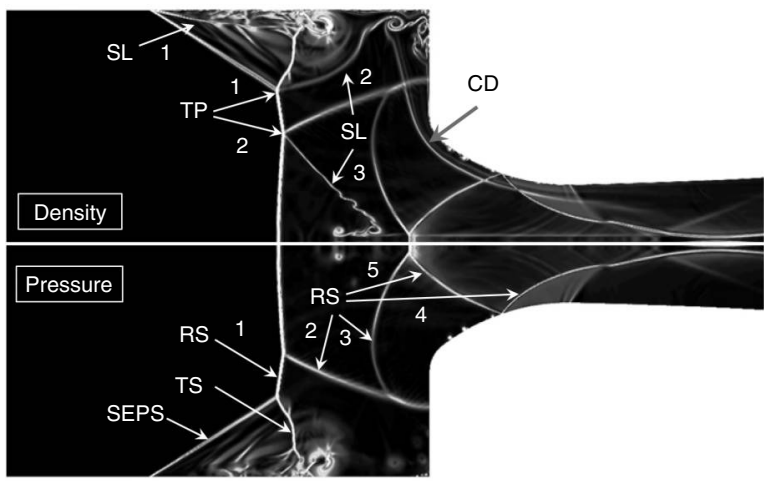

b) $t=0.497 \mathrm{~ms}$

Fig. 10 Gradients of density (upper) and pressure (lower) in the shock reflection region of model A (MS, Mach stem; TP, triple point; and TS, tail shock wave).
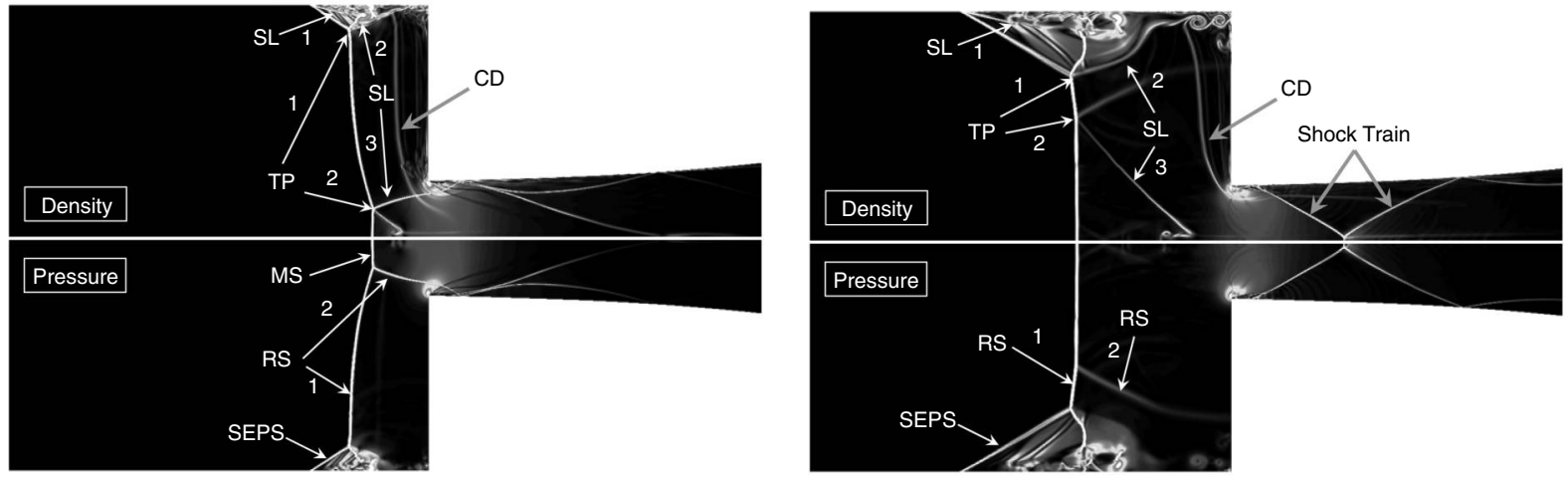

a) $t=0.237 \mathrm{~ms}$

b) $t=0.496 \mathrm{~ms}$

Fig. 11 Gradients of density (upper) and pressure (lower) in the shock reflection region of model $B$. 


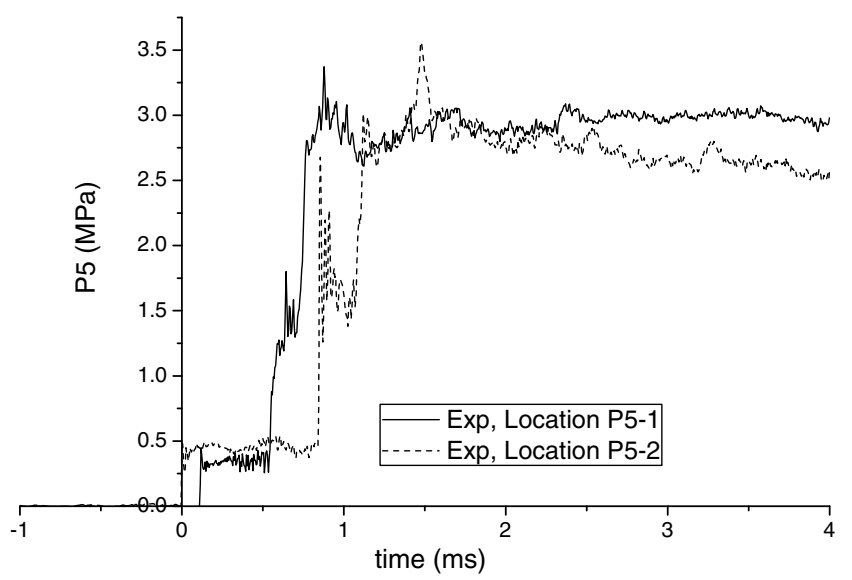

a) Experiment

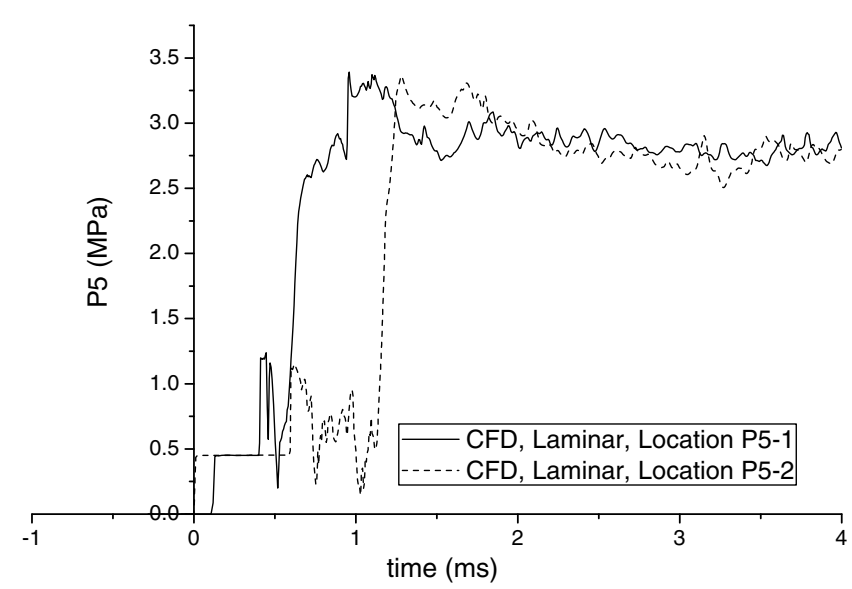

b) Model A with laminar flow by CFD

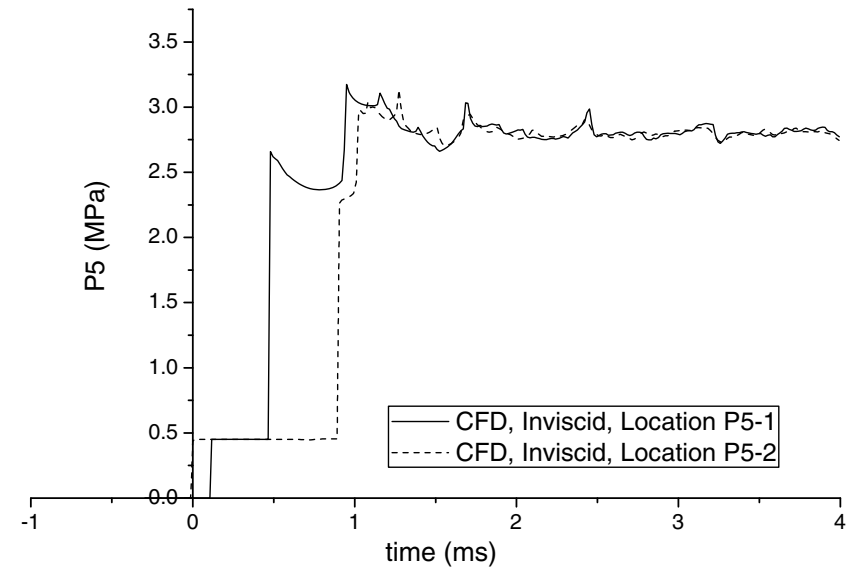

c) Model A with inviscid flow by CFD

Fig. $13 P 5$ history at $P 5-1$ and $P 5-2$ during the starting process in the case of model A.

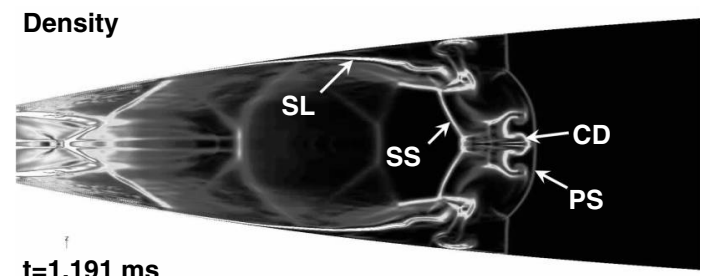

$t=1.191 \mathrm{~ms}$

a) Density gradient, $t=1.191 \mathrm{~ms}$

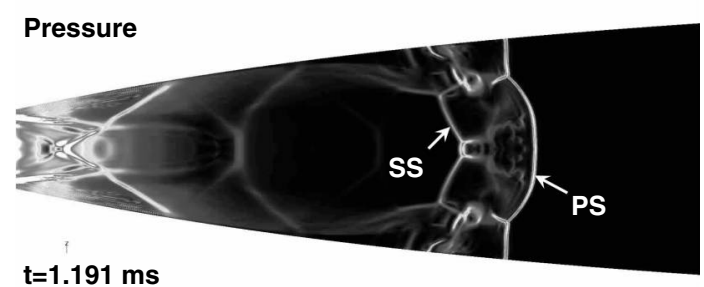

c) Pressure gradient, $t=1.191 \mathrm{~ms}$

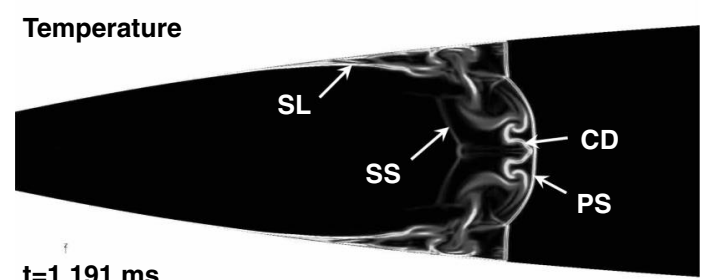

$\mathrm{t}=1.191 \mathrm{~ms}$

e) Temperature gradient, $t=1.191 \mathrm{~ms}$

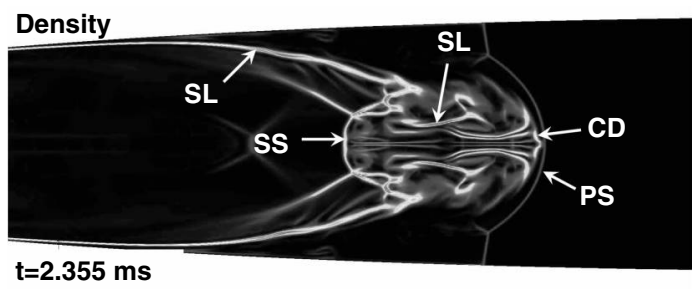

b) Density gradient, $t=2.355 \mathrm{~ms}$

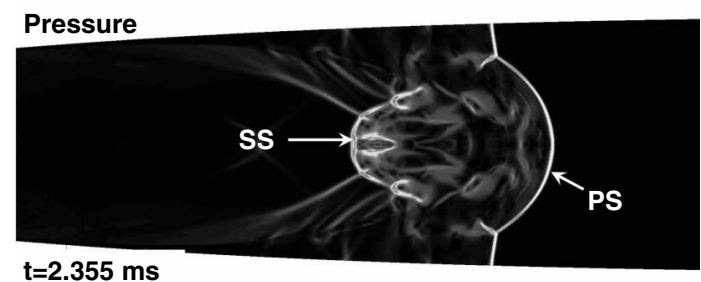

d) Pressure gradient, $t=2.355 \mathrm{~ms}$

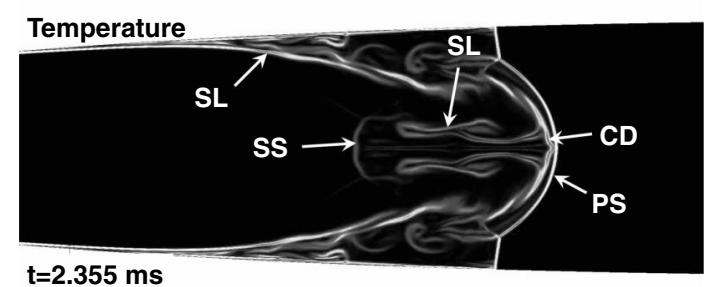

f) Temperature gradient, $t=2.355 \mathrm{~ms}$

Fig. 14 Shock structure in the nozzle (model $A$ and laminar flow). 


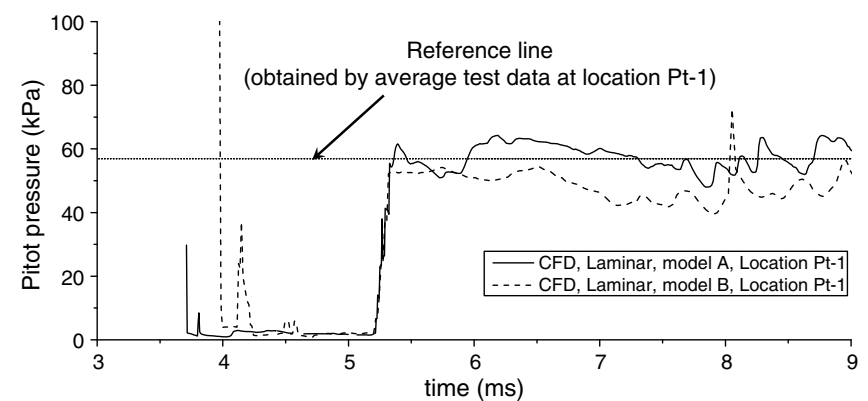

a) Laminar case

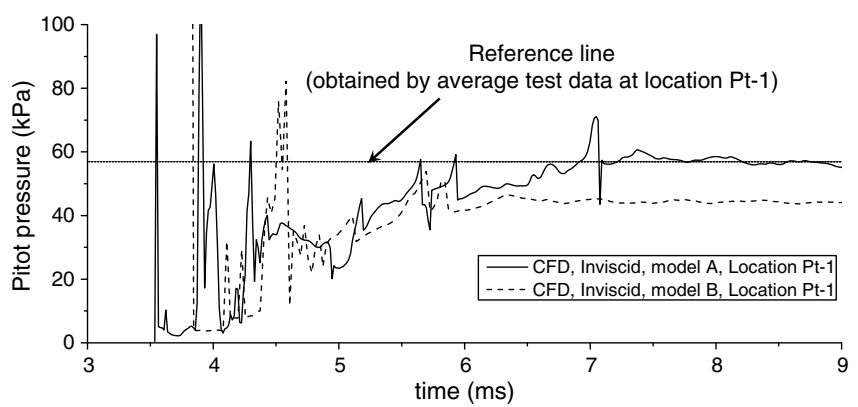

b) Inviscid case

Fig. 15 Time history of pitot pressure at location Pt-1 of nozzle exit.

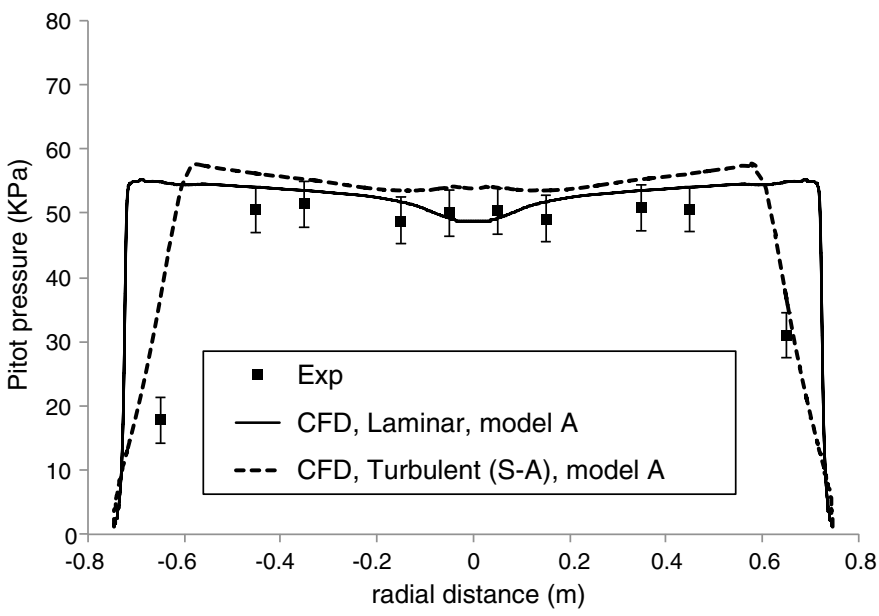

a) Pitot pressure distribution

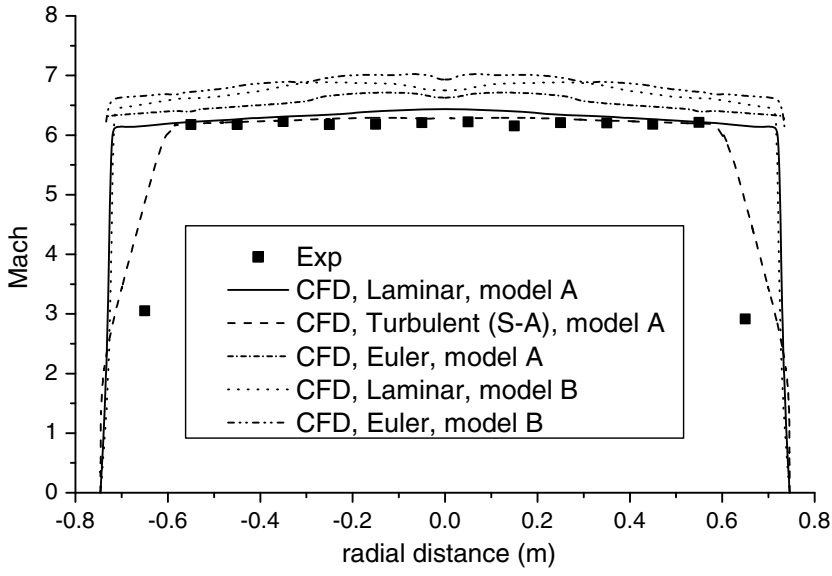

b) Mach number distribution

Fig. 16 Pitot pressure and Mach number distributions at cross section of nozzle exit.

becomes stronger as time goes on. At the beginning of the starting process, a shock train phenomenon exists and leads to a more unsteady flowfield inside the nozzle. At the same time, the SS moves away from the PS, but it still propagates downstream in the nozzle due to the accelerated supersonic nozzle flow (actually hypersonic flow near the nozzle exit). Although these phenomena were analyzed and discussed by many researchers $[\underline{1}-\underline{8}, \underline{13}, 20,21]$, the present results show the more complicated flow pattern in a large-scale shock tunnel in the case of the viscous flow. Therefore, the large nozzle has more influencing factors on the uniformity of the flowfield (flow characteristics) in the test section, such as the strength decreasing of the PS, thickening boundary layer, and strengthening interactions (e.g., shock/shock, shock/CD, shock/boundary layer, et al.).

\section{Flow Characteristics in Test Section}

The time history of the pitot pressure at the nozzle exit is presented in Fig. 15 in the cases of the laminar and inviscid flows and for two models, respectively. The larger influence, in the region between the PS (transmitted shock) and SS, is observed between the laminar and inviscid flows. The long propagating distance of the shock wave in the nozzle is considered to be the main influence factor on the more unsteady flow between the PS and SS. These influences will extend to the test section and affect the flow characteristics, such as Mach number distribution in the radial and axial directions, respectively, in the test section.

Additionally, model B was calculated and compared with model A. Obviously, the pitot pressure of model B is lower than the case of model A in the relatively stable stage (quasi-steady flow) (see Fig. 15).

Figure 16a shows the pitot pressure distribution (the averaged data at the quasi-steady state) at the cross section of the nozzle exit in the laminar case of model A. The key feature of the pitot profile is that the profiles are all reasonably flat in the test core region. However, the boundary layer obtained by CFD laminar simulation is thin and, consequently, the computed pressure near the wall is higher than the test data. To evaluate the effects of the turbulent boundary layer, the steady simulation was carried out using the Spalart-Allmaras (S-A) turbulence model in the case of model A only. The boundary layer obtained by turbulent simulation has better agreement with test data. However, the pitot pressure in the test core region is higher than the test data and laminar result. Figure $16 \mathrm{~b}$ shows Mach number distributions in the inviscid and viscous cases for two models at the nozzle exit. It is easy to see that the laminar result of model A is closer to the measurement data in the test core region. The turbulent result also shows good agreement with test data. However, the test result shows a thicker boundary layer. The reason for this difference is currently unknown. Figure 17 shows Mach number distribution

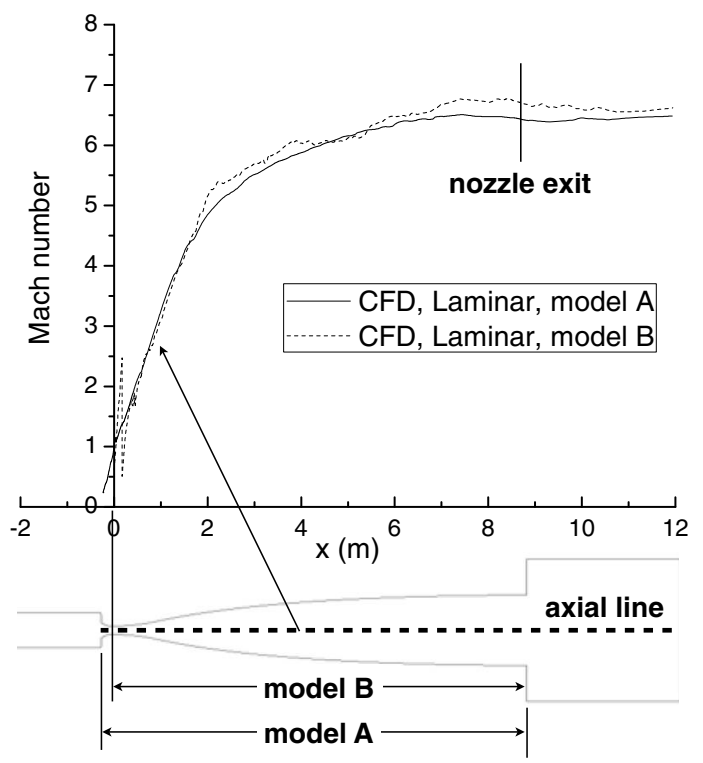

Fig. 17 Mach number distribution along nozzle axial line. 
along the nozzle axial line in models A and B. Similarly, the more inhomogeneous flow along the nozzle axial direction is seen in the case of model B, especially near the nozzle throat. The shock train that occurs in the throat region is considered to cause more unsteady flow.

\section{Conclusions}

This paper described the shock reflection and the nozzle starting processes encountered in a long-test-duration hypersonic shock tunnel. Two models were employed for seeing the effect of the throat structure on flow structure in the shock tube and on the flow uniformity in the test section. The simulation of the nozzle starting process in the case of model A (with contraction case) has a good agreement with the experimental data (e.g., the incident shock speed, pitot pressure, and Mach number distribution at the nozzle exit). It indicates that the suitable contraction section is important in the design of the large-scale nozzle, where a feasible structural design will accelerate test gas to the quasi-steady state and shorten the startup time of the nozzle flow. Obviously, based on the present results, if model B is used for the JF12 shock tunnel, then 1) the speed of the SS and PS becomes slower than model A; 2) the strength of the RS decreases due to a shock train phenomenon; 3) the pitot pressure in the test section becomes lower; 4) the Mach number in the test section is higher than model $\mathrm{A}$; and 5) the flow becomes more inhomogeneous in the nozzle and test section.

In addition, the Spalart-Allmaras turbulent model was used for checking the flowfield in the boundary layer, and the present in-house code shows good results (only steady calculation). The unsteady laminar case was analyzed in detail to assess and understand the experimental data, especially flow phenomena in the reservoir. The limitation of the present laminar simulation was also discussed for the difference between the experimental and CFD results, where the computed boundary layer is thinner than the experimental data in the laminar simulation. In future work, unsteady turbulent simulation will be carried out and the boundary layer in the shock tube will be simulated with the full size of the driven section. The nonequilibrium process will be considered for examining the starting process of the large-scale nozzle in the high-enthalpy case.

\section{Acknowledgments}

This work was supported by the National Natural Science Foundation of China (project number 11302232). Wei Zhao and Chaokai Yuan carried out the experiments in this study. The authors would like to thank them for their cooperation.

\section{References}

[1] Saito, T., and Takayama, K., "Numerical Simulations of Nozzle Starting Process," Shock Waves, Vol. 9, No. 2, 1999, pp. 73-79. doi: $10.1007 / \mathrm{s} 001930050141$

[2] Smith, C., "The Starting Process in a Hypersonic Nozzle," Journal of Fluid Mechanics, Vol. 24, No. 4, 1966, pp. 625-640. doi: $10.1017 / \mathrm{S} 0022112066000880$

[3] Amann, H., "Experimental Study of the Starting Process in a Reflection Nozzle," Physics of Fluids, Vol. 12, No. 5, 1969, pp. I-150. doi:10.1063/1.1692597
[4] Igra, O., Falcovitz, J., Reichenbach, H., and Heilig, W., "Experimental and Numerical Study of the Interaction Between a Planar Shock Wave and a Square Cavity," Journal of Fluid Mechanics, Vol. 313, No. 1, 1996, pp. 105-130. doi:10.1017/S0022112096002145

[5] Prodromou, P., and Hillier, R., "Computation of Unsteady Nozzle Flows," Shock Waves, edited by Takayama, K., Springer, Berlin, 1996, pp. 1113-1118.

[6] Tocarcik-Polsky, S., and Cambier, J., "Numerical Study of Transient Phenomena in Shock Tunnels," AIAA Journal, Vol. 32, No. 5, 1994, pp. 971-978. doi: $10.2514 / 3.12082$

[7] Jacobs, P. A., "Simulation of Transient Flow in a Shock Tunnel and a High Mach Number Nozzle," NASA CR-187606, 1991.

[8] Mouronval, A.-S., and Abdellah, H., "Numerical Study of the Starting Process in a Supersonic Nozzle," Journal of Propulsion and Power, Vol. 21, No. 2, 2005, pp. 374-378. doi: $10.2514 / 1.6122$

[9] Candler, G. V., and Perkins, J. N., "Effects of Vibrational Nonequilibrium on Axisymmetric Hypersonic Nozzle Design," AIAA Paper 1991-0297, Jan. 1991.

[10] Candler, G.V., "Hypersonic Nozzle Analysis Using an Excluded Volume Equation of State," AIAA Paper 2005-5202, June 2005.

[11] Maclean, M., Holden, M., Wadhams, T., and Parker, R., "A Computational Analysis of Thermochemical Studies in the LENS Facilities," AIAA Paper 2007-0121, 2007.

[12] MacLean, M., Dufrene, A., Wadhams, T., and Holden, M., "Numerical and Experimental Characterization of High Enthalpy Flow in an Expansion Tunnel Facility," AIAA Paper 2010-1562, Jan. 2010.

[13] Jiang, Z., and Yu, H., "Experiments and Development of Long-TestDuration Hypervelocity Detonation-Driven Shock Tunnel (LHDst)," AIAA Paper 2014-1012, 2014.

[14] Blazek, J., Computational Fluid Dynamics: Principles and Applications, 2nd ed., Elsevier Science, Oxford, 2005, pp. 448-449.

[15] Jameson, A., and Baker, T. J., "Solution of the Euler Equations for Complex Configurations," AIAA Paper 1983-1929, 1983.

[16] Shima, E., and Kitamura, K., "On New Simple Low-Dissipation Scheme of AUSM-Family for All Speeds," AIAA Paper 2009-136, 2009.

[17] Kitamura, K., and Shima, E., "Improvements of Simple LowDissipation AUSM Against Shock Instabilities in Consideration of Interfacial Speed of Sound," Proceedings of the Fifth European Conference on Computational Fluid Dynamics ECCOMAS CFD, Lisbon, Portugal, June 2010, Paper 1283.

[18] Spalart, P. R., and Allmaras, S. R., "A One-Equation Turbulence Model for Aerodynamic Flows," AIAA Paper 1992-0439, Jan. 1992.

[19] Tamura, Y., and Fujii, K., "Visualization Method for Computational Fluid Dynamics with Emphasis on the Comparison with Experiments," Proceedings of the 12th International Conference on Numerical Methods in Fluid Dynamics, Vol. 371, edited by Morton, K. W., Lecture Notes in Physics, Univ. of Oxford, Oxford, July 1990, pp. 406-410.

[20] Mark, H., "The Interaction of a Reflected Shock Wave with the Boundary Layer in a Shock Tube," NACA TM-1418, 1958.

[21] Li, J., "Investigation into Essential Problems of Detonation-Driven High Enthalpy Shock Tunnels," Ph.D. Thesis, Inst. of Mechanics, Chinese Academy of Sciences, Beijing, 2007.

G. V. Candler Associate Editor 